\title{
CONSUMO DE VERDURAS Y FRUTAS EN CENTROS \\ DE SALUD Y SUPERMERCADOS, DE LA REGIÓN METROPOLITANA DE CHILE: PROGRAMA 5 AL DÍA
}

\section{VEGETABLES AND FRUITS CONSUMPTION IN HEALTH CENTRES AND SUPERMARKETS IN THE CHILEAN METROPOLITAN REGION: 5 A DAY PROGRAM}

\author{
Isabel Zacarías H. (1), Lorena Rodríguez O. (2), Lydia Lera M. (1), Renée Hill D. (1),
}

Alejandra Domper R. (1), Daniela González A. (1)

(1) Instituto de Nutrición y Tecnología de los Alimentos, INTA, Universidad de Chile, Santiago, Chile

(2) Ministerio de Salud, Chile

\begin{abstract}
Objective: To evaluate the consumption frequency of vegetables and fruits and the knowledge of the ì a day $\hat{\imath}$ message in users of supermarkets and Public Health Centers to be utilized as a baseline to plan a bimodal controlled intervention for the promotion of fruits and vegetables consumption. Individuals and methods: A frequency consumption survey, the behavioral change stage and the message understanding was applied to 1,897 adults aged between 17 and 60 years in the urban area of the Metropolitan Region. Results: $58.8 \%$ stated that they ate between 1-2 portions/day, 33.5\% between 3-4,91.2\% indicated that they had maintained this consumption for over six months and $7.7 \%$ complied with the recommendation of $i 5$ a dayî; only $9.4 \%$ knew the message. There were no significant differences among the intervention groups except in the educational level and the number of daily consumption portions. Conclusions: The consumption of vegetables and fruits was under the recommendation in all the studied groups and there is a lack of knowledge regarding the i5 a dayî promotional message.
\end{abstract}

Key words: 5 a day; fruit and vegetables; stage of change; eating habits; food frequency.

Este trabajo fue recibido el 30 de Octubre de 2008 y aceptado para ser publicado el 20 de Marzo de 2009.

\section{INTRODUCCIÓN}

Tanto en Chile, como en otros países, el exceso de peso presenta una prevalencia alta y creciente desde los primeros años de vida. A modo de ejemplo se observa un aumento de obesidad en escolares de primero básico de $17,3 \%$ en el año 2004 a 19,4\% en el 2006 (1), teniendo en Chile una meta de llegar a 12\% para el año 2010. Según el Estudio "Carga de Enfermedad y Carga Atribuible" (2), las enfermedades no transmisibles constituyen el $84 \%$ de los años de vida perdidos ajustados por discapacidad. El consumo de alcohol, el exceso de peso y la hipertensión, son los principales factores de riesgo atribuibles a los años de vida perdidos por discapacidad. Según la última encuesta de salud de la población chilena (año 2003) realizada en 3619 adultos mayores de 17 años $50 \%$ de la población tiene riesgo alto o muy alto de enfermedad cardiovascular, el porcentaje de adultos con hipertensión alcanza a $33.7 \%$ de la muestra, mientras que $60 \%$ tiene exceso de peso u obesidad (3).

Considerando los antecedentes dados y los factores involucrados en esta realidad, entre los cuales destaca la alimentación y la actividad física, queda de manifiesto la necesidad de continuar realizando intervenciones para promover e instalar, una alimentación saludable y actividad física periódica a nivel poblacional.

El año 2004, los autores de la presente investigación se sumaron a la iniciativa internacional de promover el consumo de frutas y verduras a través del "Programa 5 al Día" para lo cual se han realizado una serie de actividades intersectoriales, de difusión del mensaje " 5 al Día" y consolidación de un grupo de trabajo, que en el año 2006 pasó a ser la Corporación 5 al Día Chile $(4,5)$. 
Existe a nivel internacional suficiente investigación científica que demuestra los efectos beneficiosos para la salud del consumo de frutas y verduras $(6,7)$. Esta recomendación en América Latina en general y en Chile en particular, a pesar de estar incluidas en las guías de alimentación, no se han hecho suficientemente efectivas $(8,9)$. Esto se debe en parte a la falta de estrategias efectivas de difusión y a otras medidas que estimulen el consumo de frutas y verduras. En este sentido el presente estudio constituye un aporte y a la vez un incentivo para que diferentes instituciones se comprometan a realizar actividades en pos de una alimentación saludable, con énfasis en la promoción del consumo de frutas y verduras.

Chile no cuenta con una encuesta nacional de consumo alimentario, los escasos datos disponibles respecto de consumo de frutas y verduras, provienen de la Encuesta de Calidad de Vida, MINSAL 2000 y $2006(10,11)$ y otras fuentes de información en grupos específicos (1214), demuestran en general un consumo de verduras y frutas bajo la recomendación internacional en todos los grupos estudiados hecho que apoya la necesidad de planificar nuevas estrategias que fomenten el consumo de estos alimentos.

El objetivo de la presente investigación fue evaluar la frecuencia de consumo de verduras y frutas y el conocimiento del mensaje "5 al día", en dos estratos, usuarios de Supermercados y usuarios de Centros de Salud Pública, para ser utilizados como línea base para planificar una intervención bimodal, controlada, para la promoción del consumo de frutas y verduras.

\section{SUJETOS Y MÉTODOS}

Se trata de un estudio descriptivo de corte transversal.

Sujetos: Se encuestaron 1897 adultos sanos entre 17 y 60 años, de la ciudad de Santiago, Chile, en Noviembre y Diciembre del año 2006, que estuvieran dispuestos a dar su número de teléfono, de ambos sexos, los que fueron seleccionados al azar, proporcionalmente al sexo y la edad, hasta completar una proporción definida por los autores, para sexo $70 \%$ de mujeres y para edad de $70 \%$ entre 30 y 60 años. A estos sujetos se les preguntó al inicio de la aplicación del instrumento si habían contestado anteriormente la encuesta con el objeto de excluirlos y evitar el posible cruce de información entre los diferentes grupos de intervención que se describen a continuación. Todos los sujetos firmaron un consentimiento informado, aprobado por el comité de ética del INTA.

La muestra fue tomada en dos tipos de establecimientos (o estratos), de nivel socioeconómico bajo y medio bajo, 9 Supermercados (comunas de Puente
Alto, La Florida, El Bosque, La Cisterna, Pedro Aguirre Cerda, Santiago, Quinta Normal y Renca) y 9 Centros de Salud Pública (comunas de Peñalolen, Macul, Cerrillos y Maipú). En cada estrato se constituyeron 3 grupos para una posterior intervención, como se explica a continuación:

- Grupo 1: Para una intervención con entrega de material educativo más explicación verbal. Este grupo se obtuvo de tres Supermercados (Comunas de La Cisterna, El Bosque y Pedro Aguirre Cerda) y de dos Centros de Salud Pública (comunas de Macul y Peñalolén).

- Grupo 2: Para una intervención con entrega de material educativo. Este grupo se obtuvo de dos Supermercados (Comunas La Florida y Puente Alto) y de 3 Centros de Salud Pública (Comuna de Peñalolén).

- Grupo Control: Sin intervención. Este grupo se obtuvo de 3 supermercados (Comunas de Santiago, Quinta Normal y Renca) y de 3 Centros de Salud Pública (Comunas de Maipú y Cerrillos).

El tamaño muestral se obtuvo considerando una potencia del $90 \%$ y un nivel de significancia de 5\%, en base a los resultados de Williams-Piehota et al (15); lo que dio un $\mathrm{n}$ de 157 personas por grupo de intervención, en cada estrato (supermercado y consultorio), lo que hace un total de 942 personas. Para conformar la línea base se ajustó el tamaño muestral a las pérdidas, según antecedentes de estudio previos (16).

Método: Se aplicó una encuesta adaptada y validada (17) que incluía: frecuencia de consumo de verduras y frutas; preguntas para determinar etapa de cambio de conducta alimentaria según Proshaska (18) y preguntas para determinar conocimiento del mensaje 5 día. Se definió que una porción de fruta o verdura corresponde aproximadamente a 80 gramos, de tal forma que la recomendación de la OMS de $400 \mathrm{~g}$ diarios, corresponden a 5 porciones. Esta encuesta se aplicó en dos tipos de establecimientos, usuarios de Centros de Salud y usuarios de Supermercados, de 12 comunas de la zona urbana de la Región Metropolitana de Santiago de Chile. Las encuestas fueron aplicadas por estudiantes de 3er y 4to año de la carrera de Nutrición y Dietética de la Universidad de Chile, debidamente capacitadas.

Análisis estadístico: Se calculan las frecuencias relativas expresadas en porcentaje. Las comparaciones de consumo de verduras y frutas, de conocimiento del mensaje5 al día y la intención de aumentar el consumo de verduras y frutas se realizaron con el test de Chi. Para el análisis estadístico de los datos se utilizó el programa Stata 10.0 (19). 


\section{RESULTADOS}

En la tabla 1 se muestran las características de la población encuestada, según estrato y grupo de intervención. Se puede observar que $70,5 \%$ corresponde al sexo femenino, $32,3 \%$ son personas entre 17 y 30 años y $67,7 \%$ entre 30 y 60 años. En relación al número de personas por grupo de intervención, se observa que cada grupo quedó constituido por alrededor de 630 personas, siendo el total de la muestra 1897. En cuanto a la escolaridad se encontró $53,9 \%$ con enseñanza media completa y $31,8 \%$ con educación superior, solo 3 personas indicaron analfabetismo (se omitió de la tabla). No se encontraron diferencias significativas entre los grupos de intervención, ni por establecimiento, en relación a edad y sexo. Se encontraron diferencias significativas en el nivel de escolaridad, siendo mayor en las personas que asisten a supermercados y en el grupo de intervención 2.

\section{TABLA 1}

\section{Descripción de la muestra según tipo de intervención y establecimiento}

\begin{tabular}{|c|c|c|c|c|c|c|c|c|}
\hline \multirow{4}{*}{$\begin{array}{l}\text { Consultorio } \\
\text { Sexo }^{1}\end{array}$} & \multicolumn{2}{|c|}{ Grupo 1} & \multicolumn{2}{|c|}{ Grupo 2} & \multicolumn{2}{|c|}{ Control } & \multicolumn{2}{|c|}{ Total } \\
\hline & \multirow[t]{2}{*}{ n } & $\%$ & \multirow[t]{2}{*}{$\mathrm{n}$} & \multirow[t]{2}{*}{$\%$} & \multirow[t]{2}{*}{$\mathrm{n}$} & \multirow[t]{2}{*}{$\%$} & \multirow[t]{2}{*}{ n } & \multirow[t]{2}{*}{$\%$} \\
\hline & & & & & & & & \\
\hline & \\
\hline Femenino & 229 & 72,9 & 227 & 70,9 & 227 & 71,8 & 683 & 71,9 \\
\hline \multicolumn{9}{|l|}{ Edad $^{2}$} \\
\hline 17 a 30 años & 109 & 34,7 & 106 & 33,1 & 106 & 33,5 & 321 & 33,8 \\
\hline 30 a 60 años & 205 & 65,3 & 214 & 66,9 & 210 & 66,5 & 629 & 66,2 \\
\hline \multicolumn{9}{|l|}{ Escolaridad $^{3}$} \\
\hline Básica & 54 & 17,4 & 92 & 29,0 & 54 & 17,1 & 200 & 21,1 \\
\hline Media & 202 & 65 & 191 & 60,3 & 207 & 65,5 & 600 & 63,4 \\
\hline Superior & 55 & 17,7 & 34 & 10,7 & 55 & 17,4 & 144 & 15,2 \\
\hline \multicolumn{9}{|c|}{ Supermercado } \\
\hline \multicolumn{9}{|c|}{ Sexo $^{4}$} \\
\hline Femenino & 219 & 69,5 & 218 & 69,0 & 218 & 69,0 & 655 & 69,2 \\
\hline \multicolumn{9}{|l|}{ Edad $^{5}$} \\
\hline 17 a 30 años & 96 & 30,5 & 98 & 31,1 & 98 & 31,1 & 292 & 30,9 \\
\hline 30 a 60 años & 219 & 69,5 & 217 & 68,9 & 217 & 68,9 & 654 & 69,1 \\
\hline \multicolumn{9}{|l|}{ Escolaridad $^{6}$} \\
\hline Básica & 24 & 7,6 & 18 & 5,8 & 24 & 8,1 & 66 & 7,2 \\
\hline Media & 160 & 50,8 & 124 & 39,7 & 123 & 41,6 & 407 & 44,1 \\
\hline Superior & 131 & 41,6 & 170 & 54,5 & 149 & 50,3 & 450 & 48,8 \\
\hline \multicolumn{9}{|c|}{ Grupo completo } \\
\hline \multicolumn{9}{|l|}{ Sexo } \\
\hline Femenino & 447 & 71,2 & 445 & 70,1 & 445 & 70,4 & 1338 & 70,5 \\
\hline \multicolumn{9}{|l|}{ Edad } \\
\hline 17 a 30 años & 205 & 32,6 & 204 & 32,1 & 204 & 32,3 & 613 & 32,3 \\
\hline 30 a 60 años & 424 & 67,4 & 432 & 67,9 & 427 & 67,7 & 1283 & 67,7 \\
\hline \multicolumn{9}{|l|}{ Escolaridad } \\
\hline Básica & 78 & 12,5 & 110 & 17,5 & 78 & 12,8 & 266 & 14,2 \\
\hline Media & 362 & 57,8 & 315 & 50,1 & 330 & 53,9 & 1007 & 53,9 \\
\hline Superior & 186 & 29,7 & 204 & 32,4 & 204 & 33,3 & 594 & 31,8 \\
\hline Total & 629 & 100 & 636 & 100 & 632 & 100 & 1897 & 100 \\
\hline
\end{tabular}


En la tabla 2 se presenta la frecuencia de consumo de frutas y verduras, según tipos de preparación (jugos, frutas enteras frescas o cocidas y verduras crudas en ensaladas o cocidas en guisos), por grupo de intervención, en el estrato Centro de Salud Público. En los 3 grupos de intervención, se observa que la forma de consumo más frecuente para frutas, es entera, ya sea frescas o cocidas, sin diferencias significativas entre ellos. Se encontró una baja frecuencia de consumo de jugos de fruta natural, en todos los grupos, sólo un tercio de los encuestados los consume entre uno a cuatro veces por semana. En relación a las verduras, un alto porcentaje de los encuestados $(61,5 \%)$ lo consume como ensalada, prácticamente a diario, no encontrándose diferencias significativas por grupo de intervención en esta forma de consumo, pero si en la forma de guisos la que es más frecuente en el grupo control. En general es mayor el consumo de verduras respecto al de frutas.

En la tabla 3 se muestra la misma información que en la tabla anterior, pero en el estrato supermercado. Se puede observar que en este grupo también la forma preferida de consumo de las frutas es entera, sea fresca o cocida, $39,7 \%$ mientras que el consumo en forma de jugo natural en la misma frecuencia, sólo lo hace $16,8 \%$

\section{TABLA 2}

Frecuencia de consumo de frutas y verduras, según tipo de preparación, en el estrato centros de salud pública, por grupo de intervención

\begin{tabular}{|c|c|c|c|c|c|c|c|c|}
\hline \multirow[t]{2}{*}{ Frecuencia } & \multicolumn{2}{|c|}{ Grupo 1} & \multicolumn{2}{|c|}{ Grupo 2} & \multicolumn{2}{|c|}{ Control } & \multicolumn{2}{|c|}{ Total } \\
\hline & $\mathbf{n}$ & $\%$ & $\mathbf{n}$ & $\%$ & $\mathbf{n}$ & $\%$ & $\mathbf{n}$ & $\%$ \\
\hline \multicolumn{9}{|c|}{ Jugos de fruta natural sin envasar ${ }^{1}$} \\
\hline Menos de $3 \mathrm{v} / \mathrm{mes}$ & 185 & 59,1 & 174 & 54,4 & 193 & 61,1 & 552 & 58,2 \\
\hline $1-4 \mathrm{v} / \mathrm{sem}$ & 092 & 29,4 & 100 & 31,2 & 085 & 26,9 & 277 & 29,2 \\
\hline $5-6 \mathrm{v} / \mathrm{sem}-1 \mathrm{v} /$ día & 024 & 7,7 & 028 & 8,8 & 027 & 8,5 & 079 & 8,3 \\
\hline 2 - 4 v/día & 012 & 3,8 & 018 & 5,6 & 011 & 3,5 & 041 & 4,3 \\
\hline Total & 313 & & 320 & & 316 & & 949 & \\
\hline \multicolumn{9}{|l|}{ Frutas frescas o cocidas ${ }^{2}$} \\
\hline Menos de $3 \mathrm{v} / \mathrm{mes}$ & 034 & 10,8 & 037 & 11,6 & 026 & 8,2 & 097 & 10,2 \\
\hline $1-4 \mathrm{v} / \mathrm{sem}$ & 116 & 36,9 & 113 & 35,3 & 110 & 34,8 & 339 & 35,7 \\
\hline $5-6 \mathrm{v} / \mathrm{sem}-1 \mathrm{v} /$ día & 081 & 5,8 & 084 & 26,3 & 084 & 26,6 & 249 & 26,2 \\
\hline 2 - 4 v/día & 083 & 26,4 & 084 & 26,3 & 090 & 28,5 & 257 & 27,0 \\
\hline 5 v/día & 000 & 0,0 & 002 & 0,6 & 006 & 1,9 & 008 & 0,8 \\
\hline Total & 314 & & 320 & & 316 & & 950 & \\
\hline \multicolumn{9}{|c|}{ Ensaladas crudas (verduras) ${ }^{3}$} \\
\hline Menos de $3 \mathrm{v} / \mathrm{mes}$ & 006 & 1,9 & 004 & 1,3 & 010 & 3,2 & 020 & 2,1 \\
\hline $1-4 \mathrm{v} / \mathrm{sem}$ & 051 & 16,3 & 052 & 16,3 & 052 & 16,5 & 155 & 16,3 \\
\hline $5-6 \mathrm{v} / \mathrm{sem}-1 \mathrm{v} /$ día & 192 & 61,3 & 200 & 62,5 & 192 & 60,8 & 584 & 61,5 \\
\hline 2 - 4 v/día & 063 & 20,1 & 064 & 20,0 & 062 & 19,6 & 189 & 19,9 \\
\hline 5 v/día & 001 & 0,3 & 000 & 0,0 & 000 & 0,0 & 001 & 0,1 \\
\hline Total & 313 & & 320 & & 316 & & 949 & \\
\hline \multicolumn{9}{|l|}{ Verduras cocidas (guisos) ${ }^{4}$} \\
\hline Menos de $3 \mathrm{v} / \mathrm{mes}$ & 61 & 19,49 & 28 & 8,78 & 26 & 8,25 & 115 & 12,14 \\
\hline $1-4 \mathrm{v} / \mathrm{sem}$ & 202 & 64,54 & 212 & 66,46 & 234 & 74,29 & 648 & 68,43 \\
\hline $5-6 \mathrm{v} / \mathrm{sem}-1 \mathrm{v} /$ día & 46 & 14,70 & 68 & 21,32 & 51 & 16,19 & 165 & 17,42 \\
\hline $2-4$ v/día & 4 & 1,28 & 11 & 3,45 & 4 & 1,27 & 19 & 2,01 \\
\hline 5 v/día & 0 & & 0 & & 0 & & 0 & \\
\hline Total & 313 & & 319 & & 315 & & 947 & \\
\hline${ }^{2} \chi^{2}=3,22 ; \mathrm{NS}$ & & & & & & & & \\
\hline
\end{tabular}


de la población encuestada; sin diferencias significativas entre los grupos de intervención. Respecto a las verduras, la forma de mayor consumo es como ensaladas crudas donde $61,1 \%$ señaló consumirlas entre 5 a 7 veces por semana, no se encontró diferencias significativas entre los distintos grupos excepto para la forma guisos, al igual que en el estrato centro de salud.

En la tabla 4 se presenta el consumo de verduras y frutas expresado en número de porciones diarias, por grupo de intervención y por tipo de establecimiento. Los valores promedio de ambos estratos indican que 58,8\% de las personas señaló consumir entre 1 a 2 porciones al día y sólo 7,7\% consume la recomendación de 5 ó más porciones al día, sin diferencias significativas entre establecimientos. Cabe hacer notar que en esta pregunta se encontraron diferencias significativas entre los distintos grupos a intervenir.

Frente a la pregunta "hace cuánto tiempo que consume este número de porciones de verduras y frutas", la mayoría de las personas $(91,2 \%)$ indicaron que lo mantiene hace mas de seis meses (cifras no incluidas en la tabla).

\section{TABLA 3}

Frecuencia de consumo de frutas y verduras, según tipo de preparación, en el estrato supermercado, por grupo de intervención.

\begin{tabular}{|c|c|c|c|c|c|c|c|c|}
\hline \multirow[t]{2}{*}{ Frecuencia } & \multicolumn{2}{|c|}{ Grupo 1} & \multicolumn{2}{|c|}{ Grupo 2} & \multicolumn{2}{|c|}{ Control } & \multicolumn{2}{|c|}{ Total } \\
\hline & $\mathbf{n}$ & $\%$ & $\mathbf{n}$ & $\%$ & $\mathbf{n}$ & $\%$ & $\mathbf{n}$ & $\%$ \\
\hline \multicolumn{9}{|c|}{ Jugos de fruta natural sin envasar ${ }^{1}$} \\
\hline Menos de $3 \mathrm{v} / \mathrm{mes}$ & 146 & 46,7 & 144 & 45,6 & 163 & 51,6 & 453 & 47,9 \\
\hline $1-4 \mathrm{v} / \mathrm{sem}$ & 99 & 31,6 & 100 & 31,6 & 101 & 32,0 & 300 & 31,8 \\
\hline $5-6 \mathrm{v} / \mathrm{sem}-1 \mathrm{v} /$ día & 52 & 16,6 & 063 & 19,9 & 044 & 13,9 & 159 & 16,8 \\
\hline 2 - 4 v/día & 14 & 4,5 & 009 & 2,9 & 008 & 2,5 & 031 & 3,3 \\
\hline 5 v/día & 02 & 0,6 & 000 & 0,0 & 000 & 0,0 & 002 & 0,2 \\
\hline Total & 313 & & 316 & & 316 & & 945 & \\
\hline \multicolumn{9}{|l|}{ Frutas frescas o cocidas ${ }^{2}$} \\
\hline Menos de $3 \mathrm{v} / \mathrm{mes}$ & 020 & 6,4 & 017 & 5,4 & 016 & 5,1 & 053 & 5,6 \\
\hline $1-4 \mathrm{v} / \mathrm{sem}$ & 091 & 28,9 & 080 & 25,4 & 084 & 26,6 & 255 & 26,9 \\
\hline $5-6 \mathrm{v} / \mathrm{sem}-1 \mathrm{v} / \mathrm{día}$ & 130 & 41,4 & 115 & 36,5 & 130 & 41,1 & 375 & 39,7 \\
\hline 2 - 4 v/día & 069 & 21,9 & 100 & 31,8 & 084 & 26,6 & 253 & 26,8 \\
\hline 5 v/día & 004 & 1,3 & 003 & 0,9 & 002 & 0,6 & 009 & 0,9 \\
\hline Total & 314 & & 315 & & 316 & & 945 & \\
\hline \multicolumn{9}{|l|}{ Ensaladas crudas ${ }^{3}$} \\
\hline Menos de $3 \mathrm{v} / \mathrm{mes}$ & 001 & 0,3 & 003 & 0,9 & 004 & 1,3 & 008 & 0,9 \\
\hline $1-4 \mathrm{v} / \mathrm{sem}$ & 056 & 17,8 & 056 & 17,7 & 056 & 17,7 & 168 & 17,8 \\
\hline $5-6 \mathrm{v} / \mathrm{sem}-1 \mathrm{v} /$ día & 200 & 63,7 & 184 & 58,2 & 194 & 61,4 & 578 & 61,1 \\
\hline $2-4$ v/día & 057 & 18,2 & 071 & 22,5 & 062 & 19,6 & 190 & 20,1 \\
\hline 5 v/día & 000 & 0,0 & 002 & 0,6 & 000 & 0,0 & 002 & 0,2 \\
\hline Total & 314 & & 316 & & 316 & & 946 & \\
\hline \multicolumn{9}{|c|}{ Verduras cocidas (guisos) ${ }^{4}$} \\
\hline Menos de $3 \mathrm{v} / \mathrm{mes}$ & 31 & 9,9 & 66 & 21,0 & 381 & 2,1 & 135 & 14,3 \\
\hline $1-4 \mathrm{v} / \mathrm{sem}$ & 215 & 68,5 & 216 & 68,6 & 206 & 65,6 & 637 & 67,6 \\
\hline $5-6 \mathrm{v} / \mathrm{sem}-1 \mathrm{v} /$ día & 62 & 19,8 & 30 & 9,5 & 66 & 21,0 & 158 & 16,8 \\
\hline $2-4$ v/día & 6 & 1,9 & 2 & 0,6 & 4 & 1,3 & 12 & 1,3 \\
\hline 5 v/día & 0 & 0,0 & 1 & 0,3 & 0 & 0,0 & 1 & 0,1 \\
\hline Total & 314 & & 315 & & 314 & & 943 & \\
\hline
\end{tabular}


En relación a la intención de aumentar el consumo de verduras y frutas, la tabla 5 muestra que en promedio, $66 \%$ de la población encuestada de los dos estratos, está planificando aumentar su consumo para el próximo mes, sin diferencias entre los estratos, pero con diferencias significativas entre los grupos de intervención, en el estrato supermercado.

Frente a la pregunta "por qué no pretende aumentar el consumo de frutas y verduras" $74,3 \%$ señaló que la principal razón es que consideran que su actual consumo es adecuado y sólo 7\% indicó que la razón es el acceso (costo, disponibilidad).
En la tabla 6 se presenta el conocimiento del mensaje "Consuma 5 porciones de verduras y frutas al día" y se observa que en los Centros de Salud Pública 7,8\% señaló conocer este mensaje y en los Supermercados 10,9\%. No se encontraron diferencias significativas por grupo de intervención.

De las personas que señalaron conocer el mensaje "5 porciones de frutas y verduras al día" $(9,4 \%)$, en promedio $44,2 \%$ señaló haberlo conocido a través de diarios y revistas, $23 \%$ en su lugar de trabajo o estudio y $15,4 \%$ mediante otras personas. Es importante señalar que en la fecha que se realizó este estudio aún no comenzaba

\section{TABLA 4}

Número de porciones de frutas y verduras, consumidas diariamente, por grupo de intervención y tipo de establecimiento

\begin{tabular}{lcccccccc}
\multicolumn{1}{r}{ Porciones por día } & \multicolumn{2}{c}{ Grupo 1 } & \multicolumn{2}{c}{ Grupo 2 } & \multicolumn{2}{c}{ Control } & \multicolumn{2}{c}{ Total } \\
& $\mathbf{n}$ & $\mathbf{\%}$ & $\mathbf{n}$ & $\mathbf{\%}$ & $\mathbf{n}$ & $\mathbf{\%}$ & $\mathbf{n}$ & $\mathbf{\%}$ \\
\hline Consultorio1 & & & & & & & & \\
$1-2$ & 197 & 62,7 & 156 & 48,8 & 223 & 70,6 & 576 & 60,6 \\
$3-4$ & 94 & 29,9 & 133 & 41,6 & 85 & 26,9 & 312 & 32,8 \\
5 y más & 23 & 7,3 & 31 & 9,7 & 8 & 2,5 & 62 & 6,5 \\
Total & 314 & & 320 & & 316 & & 950 & \\
Supermercado2 & & & & & & & & \\
$1-2$ & 141 & 44,9 & 202 & 64,1 & 196 & 62,0 & 539 & 57,0 \\
$3-4$ & 130 & 41,4 & 92 & 29,2 & 100 & 31,7 & 322 & 34,1 \\
5 y más & 43 & 13,7 & 21 & 6,7 & 20 & 6,3 & 84 & 8,9 \\
Total & 314 & & 315 & & 316 & & 945 &
\end{tabular}

\section{TABLA 5}

Intención de aumentar el consumo de frutas y verduras, según grupo de intervención, en consultorios y supermercados.

En el próximo mes

\begin{tabular}{|lccccccccc}
\hline \multicolumn{1}{c}{ Respuesta } & \multicolumn{2}{c}{ Grupo 1 } & \multicolumn{2}{c}{ Grupo 2 } & \multicolumn{2}{c}{ Control } & \multicolumn{2}{c}{ Total } \\
& n & \% & n & \% & n & \% & n & \% \\
\hline Consultorio $^{1}$ & 202 & 64,3 & 213 & 66,8 & 193 & 61,1 & 608 & 64,1 \\
Supermercado $^{2}$ & 236 & 75,2 & 208 & 65,8 & 191 & 60,4 & 635 & 67,1 \\
\hline${ }^{1} \chi^{2}=2,25 ;$ NS $\quad{ }^{2} \chi^{2}=15,8 ; \mathrm{p}<0,0001$ & & & & & & & &
\end{tabular}


en el país la campaña de promoción de "5 porciones de frutas y verduras al día".

\section{DISCUSIÓN Y CONCLUSIONES}

Los resultados del presente estudio constituyen una línea base sobre la frecuencia de consumo de verduras y frutas, conocimientos de la recomendación de consumo y evaluación de la etapa de cambio en que se encuentra la población en relación a este tema. Estos antecedentes son fundamentales para la planificación de intervenciones.

En relación a la muestra seleccionada se observa que se alcanzó la distribución por edad, sexo y tipo de establecimiento comparable en los diferentes grupos de intervención y en el grupo control. El mayor nivel de escolaridad encontrado en las personas que asisten al Supermercado servirá de antecedente a considerar para futuras intervenciones en este grupo poblacional.

Los resultados relacionados con la frecuencia de consumo de verduras y frutas en ambos tipos de establecimientos indicaron que la forma más frecuente de consumo de frutas es entera, sean frescas o cocidas y además existe una distribución homogénea por grupo de intervención. Situación semejante se observó para el consumo de verduras, en que en ambos establecimientos la forma preferida de consumo es como ensaladas crudas, existiendo una distribución semejante por grupo de intervención. El conocer el tipo de preparación preferida por la población chilena permitirá orientar las campañas comunicacionales tendientes a promover el consumo de verduras y frutas. En Colombia se ha observado que la población prefiere consumir las frutas en forma de jugos de fruta natural, hecho que ha ayudado a desarrollar campañas para promocionar su consumo, en forma exitosa (20).

El hallazgo de que en la población chilena la forma preferida de consumo de las frutas es entera, sea fresca o cocida, tiene la ventaja desde el punto de vista nutri- cional de aprovechar al máximo todos los componentes bioactivos presentes en la fruta, así por ejemplo se destaca la mejor utilización de la fibra y de la vitamina $\mathrm{C}$, la que se pierde al exponerse al aire en el proceso de extracción o el picado trozado de la fruta. Para el caso de la naranja, se ha demostrado que al masticar la fruta se mezclan los flavonoides de la corteza blanca con la vitamina $\mathrm{C}$ presente en la fruta, interactuando para potenciar la acción beneficiosa en la salud de las personas $(21,22)$.

La baja frecuencia en el consumo de 5 porciones verduras y frutas al día encontrada en el presente estudio ( $7,7 \%$ de la población encuestada), demuestra que nuestra población está claramente bajo la recomendación nacional e internacional $(23,24)$ de consumir al menos 400 gramos diarios de verduras y frutas al día, lo que en términos prácticos se traduce a cinco porciones. El hecho de haber encontrado que alrededor del $60 \%$ de la población sólo consume entre una a dos porciones al día, concuerda con datos previos publicados por MINSAL en que la cantidad promedio consumida diariamente fue de $166 \mathrm{~g} /$ persona /día. Según la misma fuente el año 2000 se encontró que sólo 47\% de la población consume verduras y frutas todos los días, en tanto que en el año 2006 se encontró que 53\% de la población consumía verduras todos los días y $47 \%$ frutas, no encontrándose cambios significativos en entre los dos periodos. En estas mismas encuestas, cabe destacar que se encontraron diferencias significativas al analizar estos resultados por regiones, probablemente en correspondencia con diferentes climas, disponibilidad y situación socioeconómica $(10,11)$.

Los resultados del presente estudio concuerdan con datos publicados por IPSOS en Chile, en una encuesta telefónica realizada en el año 2005 a 1000 personas mayores de 18 años, en la cual se encontró que $6 \%$ de las personas declaró comer más de 4 porciones de frutas y $4 \%$ más de 4 porciones de verduras, al día; $58 \%$ consume

\section{TABLA 6}

Conocimiento previo del mensaje "consuma 5 porciones de frutas y verduras al día", según grupo de intervención, en consultorios y supermercados

\begin{tabular}{|c|c|c|c|c|c|c|c|c|}
\hline \multirow[t]{2}{*}{ Respuesta } & \multicolumn{2}{|c|}{ Grupo 1} & \multicolumn{2}{|c|}{ Grupo 2} & \multicolumn{2}{|c|}{ Control } & \multicolumn{2}{|c|}{ Total } \\
\hline & $\mathbf{n}$ & $\%$ & $\mathbf{n}$ & $\%$ & $\mathbf{n}$ & $\%$ & $\mathbf{n}$ & $\%$ \\
\hline Consultorio $^{1}$ & 22 & 7,0 & 24 & 7,5 & 28 & 8,9 & 74 & 7,8 \\
\hline Supermercado $^{2}$ & 37 & 11,8 & 31 & 9,9 & 35 & 11,1 & 103 & 10,9 \\
\hline
\end{tabular}


frutas todos los días y $67 \%$ consume verduras a diario (13). Por otra parte, Olivares y col (12) al evaluar la ingesta en estudiantes de 7 a 13 años de escuelas públicas de 3 regiones del país, encontró que solo $38 \%$ consumía fruta y solo $31 \%$ verduras, todos los días.

Estudios realizados en otros países indican que sólo una minoría de la población adulta mundial, consume lo mínimo recomendado (400 g/persona/día). Se ha visto además que los países de bajo nivel de desarrollo tienen el menor consumo de verduras y frutas. Así por ejemplo, de acuerdo a la última información publicada por el Instituto Nacional del Cáncer de Brasil, sólo 4,3\% de la población consume 5 o más porciones por día (25). Pormeleau, Lock et al $(26,27)$ realizaron una extensa revisión internacional de ingesta de verduras y frutas en varios países encontrando que las ingestas mas altas en algunos países de Europa, correspondieron a un consumo promedio de $449 \mathrm{~g} /$ persona/ día. En el estudio "Pro Children" en el que participaron 15.404 niñas y niños de 9 países europeos (Austria, Bélgica, Dinamarca, Islandia, Holanda, Noruega, Portugal, España y Suiza), en el cual se entrevistó también a las madres, se encontró que sólo $27 \%$ de éstas, cumple con la recomendación de los 400 $\mathrm{g}$ diarios (28).

Para el caso de España, se encontró un inadecuado consumo de verduras y frutas en los distintos grupos etáreos estudiados; a saber: niños y jóvenes $88 \%$ no alcanza a consumir las 5 porciones al día; $56 \%$ de los adultos no cumple esta recomendación, mientras que $45 \%$ de los adultos mayores, tampoco cumple con la recomendación. Estas cifras claramente señalan que es una necesidad intervenir en una población más joven (29).

El alto porcentaje $(91,2 \%)$ de personas que indicaron en la encuesta que llevan consumiendo por más de seis meses el mismo número de porciones de verduras y frutas, nos permite deducir que este consumo ya es un hábito en estas personas.

Dado que en general el consumo de verduras y frutas, está bajo la recomendación, es importante diseñar e implementar nuevas iniciativas y estrategias más efectivas para contribuir al cambio de conducta para aumentar el consumo de verduras y frutas en la población, hecho que sin duda contribuirá a mejorar la calidad de vida.

La información obtenida de la presente investigación, en que se señala que un alto porcentaje de los encuestados $(66 \%)$ tiene intenciones de aumentar el consumo, se estima que facilitará la tarea de modificar hábitos de consumo de estos alimentos. Por otra parte, conocer que la principal razón para no tener intención de cambio en el consumo de verduras y frutas, es la falta de conocimiento de la recomendación, este hecho también debería considerarse para mejorar la efectividad de futuras intervenciones.

Conocer que la principal fuente de conocimiento del mensaje "5 al día" ha sido a través de la prensa escrita, constituye un incentivo para considerar esta vía de difusión, como efectiva para sensibilizar a la población en la importancia del consumo de verduras y frutas.

Conclusiones: el consumo de verduras y frutas estuvo bajo la recomendación en todos los grupos estudiados. Existe un desconocimiento del mensaje promocional "5 al día" en la mayoría de la población entrevistada.

\section{RESUMEN}

Objetivo: Evaluar la frecuencia de consumo de verduras y frutas y el conocimiento del mensaje " 5 al día", en usuarios de Supermercados y de Centros de Salud Pública, para ser utilizados como línea base para planificar una intervención bimodal, controlada, para la promoción del consumo de frutas y verduras. Sujetos y métodos: a 1897 adultos entre 17 y 60 años, del área urbana de la Región Metropolitana, se les aplicó una encuesta de frecuencia de consumo, etapa de cambio de conducta y conocimiento del mensaje. Resultados: $58.8 \%$ señaló consumir entre 1-2 porciones/día y 33.5\% entre 3-4. $91,2 \%$ indicó que ha mantenido este consumo por más de seis meses, 7,7\% cumple con la recomendación " 5 al día" sólo 9,4\% conocía el mensaje. No hubo diferencias significativas entre los grupos de intervención salvo en el nivel educacional y en el número de porciones de consumo diario. Conclusiones: El consumo de verduras y frutas estuvo bajo la recomendación en todos los grupos estudiados y existe un desconocimiento del mensaje promocional "5 al día".

Palabras claves: 5 al día; frutas y verduras; etapa de cambio; hábitos alimentarios; frecuencia de consumo.

Dirigir la correspondencia a:

Profesora

Isabel Zacarías $\mathrm{H}$.

Profesor Asistente

INTA - Universidad de Chile

El Líbano 5524 - Macul. Santiago, Chile

Teléfono: 9781429

E-mail: izacaria@inta.cl

Agradecimientos: Financiamiento: OPS/CDC/ILSI Proyecto: Evaluación de impacto de una campaña educacional "5 al día" para aumentar el consumo de frutas y verduras. 2005-2006. Trabajo presentado en el $14^{\circ}$ Congreso Latinoamericano de Nutrición Florianópolis Brasil, Noviembre 12 -16, 2006.

Los autores agradecen la excelente participación de las alumnas de Nutrición y Dietética de la Universidad 
de Chile, agradecen también a los Servicios de Salud Metropolitano Central y Oriente y a la cadena de Supermercados Santa Isabel por brindarnos las facilidades para realizar el trabajo en terreno.

\section{BIBLIOGRAFÍA}

1. Mapa Nutricional de Chile, Junaeb, 2006 http:// sistemas.junaeb.cl/estadosnutricionales_2007/ TablaObesidadRegion.php?ano $=2006 \&$ region $=\&$ variable $=1 \&$ comuna $=\&$ provincia $=\&$ universo $=\&$ escuelas $=\&$ orden $=\&$ col_orden $=0 \&$ brecha $=\& \operatorname{sex}$ $\mathrm{o}=\&$ are $\mathrm{a}=\&$ distribucion $=$ visitado 23 de octubre de 2008 .

2. Ministerio de Salud. Estudio de carga de Enfermedad y Carga Atribuible. Minsal 2007-2008.

3. Ministerio de Salud. Encuesta de salud de la población chilena. Santiago: MINSAL; 2003. www. minsal.cl/epidemiología.

4. Vio F, Zacarías I, González D. Implementación de un programa de promoción del consumo de frutas y verduras: Corporación 5 al día Chile. En: Olivares S, Lporati M, Villalobos P, Barría L, eds. Contribución de la política agraria al consumo de frutas y verduras en Chile: un compromiso con la nutrición y la salud de la población. Ministerio de Agricultura; INTA, Universidad de Chile; Corporación 5 al día Chile. Santiago, Chile. Abril, 2008.

5. Zacarías I, PizarroT, Rodríguez L, González G, Domper D. Programa "5 al día" para promover el consumo de verduras y frutas. Rev Chil Nutr; 2006 (33) S1:276-280.

6. WHO. Fruit And Vegetables Promotion Iniciative - report of the meeting, Geneva 25-27 August, 2003.

7. WHO/FAO. Fruit and vegetables for health. Report of a joint FAO/WHO workshop 1-3 Kobe, Japón. September 2004.

8. Molina V. Guías alimentarias en América Latina. Informe de la consulta técnica regional de las Guías Alimentarias. An Venez Nutr 21: Caracas, Venezuela, 2008.

9. Albert J. Global Patterns and Country Experiences with the formulation and implementation of Foof -Based Dietary Guidelines. An Nutr Matab 2007; 51(Suppl 2): 2-7.

10. Ministerio de salud. Encuesta Nacional de Calidad de Vida y Salud. Chile 2000.

11. Ministerio de salud. Encuesta Nacional de Calidad de Vida y Salud. Chile 2006.

12. Olivares S. Consumo de frutas y verduras en Chile. Motivaciones y barreras para alcanzar la meta de 5 porciones al día. En: Olivares S, Lporati $\mathrm{M}$,
Villalobos P, Barría L, eds. Contribución de la política agraria al consumo de frutas y verduras en Chile: un compromiso con la nutrición y la salud de la población. Ministerio de Agricultura; INTA, Universidad de Chile; Corporación 5 al día Chile. Santiago, Chile. Abril, 2008

13. IPSOS, Foods Trends Internacional: Consumo, actitudes, bienestar. Santiago, Chile. Agosto 2005.

14. Collect GFK. Encuesta de consumo de frutas y verduras en Chile. Abril, 2008

15. Williams-Piehota P, Cox A, Silvera SN, Mowad L, Garcia S, Katulak N, Salovey P. Casting health messages in terms of responsibility for dietary change: increasing fruit and vegetable consumption. J Nutr Educ Behav. 2004 May-Jun;36(3):114-20.

16. Domper, Alejandra, Zacarías H., Isabel, Olivares C., Sonia et al. Evaluación de un programa de información en nutrición al consumidor. Rev Chil Nutr 2003;.30 (1):43-51.

17. Five a day for Better Health Program. Monograph. ( NIH, NCI). USA 1999.

18. Prochaska J, Velicer W, Rossi J, Goldstein M, Marcus B et al. Stages of changes and decisional balance for twelve problem behavior . Health Psychol 1994; 13: 39-46.

19. (StataCorp. 2007. Stata Statistical Software: Release 10. College Station, TX: StataCorp LP.).

20. Prada G, Dubeibe-Blanco L, Herrán O, HerreraAnaya M. Evaluación del impacto de un ensayo comunitario sobre el consumo de frutas y verduras en Colombia. Salud Pública Mex 2007;49:11-19.

21. Fondo Mundial para la investigación del cáncer/ Instituto estadounidense de investigación del cáncer. Alimentos, nutrición, actividad física y la prevención del cáncer: una perspectiva mundial. Washington, DC: AICR, 2007

22. Clarisse M, Giusti V. Fruit and vegetable beverages: advantages and disadvantages. Rev Med Suisse, 2008;4(151):829-33.

23. INTA, Ministerio de Salud. Guías para una vida saludable, Guías alimentarias Actividad Física y Tabaco. Andros Impresores, Santiago, Chile. 2005.

24. Report of a join WHO/FAO expert consultation. Diet, Nutrition and the prevention of chronic diseases. WHO Technical Report series 916, Geneva 2003.

25. Marchioni D. Nutrición y Cáncer. En: Zacarías I, Olivares S, Schuartz M, Rodríguez L. Eds. 4to Congreso Panamericano de Promoción del consumo de verduras y frutas. Actas del Congreso, pp 19. Agosto 2008.

26. Pomerleau J, Lock K, McKee M, Altmann DR. The 
challenge of measuring global fruit and vegetable intake. J Nutr 2001;134:1175-1180.

27. Lock K, Pomerleau J, Causer L, Altmann DR, McKee M. The global burden of disese attributable to low consumption of fruit and vegetables: implications for the global strategy on diet. Bulletin of the World Health Organization. February 2005, 83(2).

28. Wolf A, Yngve A, Elmadfa I, et al. Fruit and veg- etables intake of mothers of 11-year-old children in nine european countries: The Pro children Cross-sectional Survey. Ann Nutr Metab 2005; 49:246 - 254.

29. Arancetra J, Pérez C, Serra L. Consumo de frutas y verduras en España. En: Arancetra J, Pérez C, Eds. Frutas, verduras y salud. Editorial ELSEVIER, Barcelona, España, 2006. 